Témoigner Témoigner. Entre histoire et mémoire

Getuigen Revue pluridisciplinaire de la Fondation Auschwitz

$124 \mid 2017$

La musique dans les camps

\title{
Les orchestres dans le système concentrationnaire nazi : fonctions et réception
}

Orkesten in de naziconcentratiekampen: functie en receptie

\section{Amaury du Closel}

\section{(2) OpenEdition}

1 Journals

\section{Édition électronique}

URL : https://journals.openedition.org/temoigner/5754

DOI : $10.4000 /$ temoigner.5754

ISSN : 2506-6390

Éditeur :

Éditions du Centre d'études et de documentation Mémoire d'Auschwitz, Éditions Kimé

Édition imprimée

Date de publication : 2 avril 2017

Pagination : 98-112

ISBN : 978-2-930953-00-7

ISSN : 2031-4183

Référence électronique

Amaury du Closel, «Les orchestres dans le système concentrationnaire nazi : fonctions et réception », Témoigner. Entre histoire et mémoire [En ligne], 124 | 2017, mis en ligne le 30 novembre 2021, consulté le 01 décembre 2021. URL : http://journals.openedition.org/temoigner/5754 ; DOI : https://doi.org/ 10.4000/temoigner.5754 


\section{Les orchestres dans le système concentrationnaire nazi : fonctions et réception}

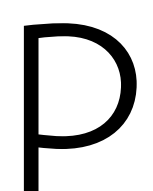

hénomène aujourd'hui largement connu, l'existence et la place de la musique - et donc dorchestes - dans lescanps nazis ne furentadnices comme réalité qu’à pchestres - dans les camps nazis ne furent admises le contexte particulier de son exercice semblait auparavant dépasser les limites de l'entendement. La multiplication des témoignages, dont certains parurent dès 1946, provenant notamment de plusieurs musiciennes ayant appartenu
àl'orchestre des femmes d'Auschwitz, dirigé par la violoniste Alma Rosé (1906-1944), permit d'en confin permit den contente cadre de cette brevve étude : nous définissons conme systène concentrationnaire nazi les divers centres de détention (camps de concentration, ghettos, prisons) créés par le Troisième Reich à partir de 1933 jusquà la fin de la Seconde Guerre mondiale, pourinterner et faire disparaître des opposants politiques, exploiter la force de travail de résidents des pays conquis et de certains prisonniers de guerre non protégés par la Convention de Genève, et à partir de 1941, d'exterminer des groupes spécifiques.

Il se distingue des camps de prisonniers inhérents à l'état de guerre, et soumi à l'époque à la Convention de Genève de 1929 qui en organisait le fonctionnement et donnait des garanties aux prisonniers. On ne trouvera donc rien dans ces lignes et donnait des garanties aux prisonniers. On ne trouvera donc rien dans ces ligne se rapportant par exemple à Olivier Messiaen ou à d'autres de ses confrères qui composèrent ou pratiquèrent la musique dans les stalags ou les oflags.

En revanche, la place de la musique dans ce système concentrationnaire n'est guère différente de celle que l'on retrouve dans tous les contextes de privation de liberté, quels qu'ils soient, du Goulag aux prisons chiliennes. Sa présence est évoquée dès la Bible dans le Psaume 137 qui évoque la déportation du peuple hébreu à Babylone après la victoire de Nabuchodonosor II au VI ${ }^{e}$ siècle avant notre ère

Sur les bords des fleuves de Babylone,

Nous étions assis et nous pleurions, en nous souvenant de Sion.

Aux saules de la contreé, nous avions suspendu nous nous nemandaient des chants,

Et nos oppresseurs de la joie: Chantez-nous quelques-uns des cantiques de Sion!
La dimension particulière que confère au système concentrationnaire nazi son rôle dans la Shoah, et dans le Porajmos des Roms et des Sintis, ajoute une perspective nouvelle au phénomène. Parce qu'elle accompagne la mort programmée de peuples entiers, la musique prend dans ce contexte une place symbolique que vient renforcer l'accent mis sur son usage dans le camp-ghetto de Theresienstadt par la propagande nazie. Son développement exceptionnel, la fonction qu'y jouèrent les bourreaux pris à titre individuel, et non comme représentants du pouvoir, sont symptomatiques et origines, le régime nazi s'est employé à contrôler et à instrumentaliser.

\section{QUELS ORCHESTRES}

\section{Création et développement}

La création et le développement d'un orchestre suit un processus relativement analogue d'un camp à l'autre. A l'origine se trouve souvent l'initiative d'un petit groupe de musiciens, d’abord clandestin, puis progressivement autorisé par la SS.

Peu de temps après la création du camp de Buchenwald en 1937, les SS forment un petit orchestre de musiciens tziganes, d'un niveau très médiocre, aucun de ses membres n'ayant de formation musicale et instrumentale. Ce sont encore les Tziganes qui formentà Mauthausen un ensemble dirigé par l'accordéoniste homosexuel Wilhelm Heckmann. Cet ensemble jouait notamment pour les petites fêtes des «Prominente» (prisonniers privilégiés) et des SS à la maison close du camp.

Au camp de Dachau, ouvert dès 1933, le premier ensemble musical fut formé par le compositeur Herbert Zipper (1904-1997) en 1938, d'abord clandestinement, puis toléré par les autorités du camp. En 1940, un groupe de détenus tchèques obtinrent l'autorisation de constituer un orchestre de salon, qui donnait des concerts de musique légère pour divertir les Kapos et les SS.

Avec l'élargissement des camps et l'arrivée de prisonniers des territoires conquis, les SS décidèrent d'agrandir les orchestres et d'en développer le rôle pour mainles SS décidèrent d'agrandir les orchestres et d’en développer le rôle pour main-
tenir le moral des prisonniers. C'est lachat de nouveaux instruments début 1941 tenir le moral des prisonniers. C'est l'achat de nouveaux instruments début 1941
- principalement des cuivres - et l'arrivée du tchèque Vlastimil Louda, arrêté pour - principalement des cuivres - et l'arrivée du tchèque Vlastimil Louda, arrêté pour
faits de résistance, comme chef de l'orchestre qui marquèrent la restructuration de l'orchestre de Buchenwald au début de 1942. Ses 32 membres portaient un uniforme spécial. En 1945, l'orchestre comptait environ 120 musiciens (dont 32 vents et 84 cordes) pouvant se diviser en divers sous-groupes, dont un ensemble de cuivres.

Au début de 1941, le commandant du camp de Dachau décida la création d'un véritable orchestre, dont le répertoire intégra rapidement quelques classiques favoris conme la deuxienne Rhapsodie hongroise de Liszt, les ouvertures d'Orphée aux Enfers d'Offenbach, La Pie voleuse de Rossini, ou Poète et Paysan de Suppé. Les 
arrangements étaient réalisés par le clarinettiste Josef Ulc. Fut également créé à l'automne 1941 un ensemble de cuivres. Parallèlement à ces formations protégées par le commandant du camp existait un orchestre à cordes dirigé par Piet van den Hurk - chef de l'orchestre de la Radio chrétienne hollandaise - au programme duquel figuraient des œuvres de Giordani, Haendel, Mozart, Beethoven et Grieg, et qui donna également le Concerto pour violon de Mendelssohn sous un faux nom de compositeur pour éviter la censure des autorités du camp. À Mauthausen, l'orchestre lui-même se développa à partir de l'ordonnance d'aôt 1942 et à la faveur de la livraison de se développa à partir de lordonnance d'août 1942 et à la faveur de la livraison de nombreux instruments dans les semaines qui suivirent. Il comptait une trentaine tard, avec l'arrivée d'une vingtaine de membres de la Philharmonie de Varsovie.

Ce processus se répète dans les camps de Flossenbürg - où la «Lagerkapelle 作 1944 de quarante à cinquante musiciens -, ou Neuengamme don l'orchestre comprenait environ 25 musiciens originaires de France, Italie, Danemark, Pologne, Belgique, Tchécoslovaquie et Allemagne. De 1942 à 1944, un second orchestre fut formé. Plus important que le précédent - il comptait de 60 à 80 musiciens -, il eut plusieurs chefs, dont le compositeur et homme de théâtre tchèque

Pendant cette période, on ne sabrat sousestimer lêtedesconm camps dans le développement de «leur» orchestre : la création de celui de Neuengamme l'avait été sur ordre du commandant du camp Martin Weiss, très impressionné par sa visite à Auschwitz où la SS venait de créer un premier orchestre. Le Kommando de musique de Natzweiler-Struthof est aussi creé en 1942 par le commandant du camp Josef Kramer, probablement sur le modèle de l'orchestre du camp d'Auschwitz, où il avait servi en 1941, et dont il deviendra le commandant en 1944. Kramer semb ou

commandant dines pour les trompettes de ses musiciens.

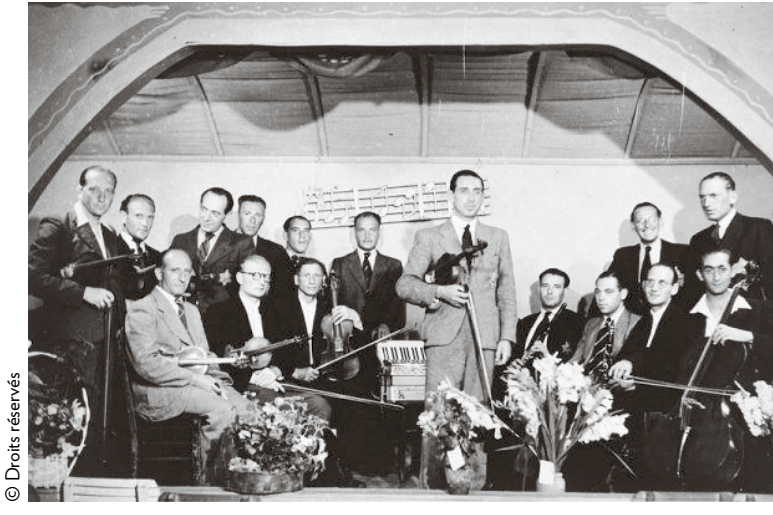

Le camp de Westerbork avait été à l'origine établi en 1939 par les autorités hollandaises pour accueilli les réfugiés juifs fuyant le régime nazi. Sous la domination nazie, le camp se transforma en 1942 en camp de transit pour ceux qui s'y trouvaient déjà ainsi que pour les Juifs hollandais. Administré par un Conseil juif, Westerbork connut une vie culturelle très développée qui se poursuivit quand les SS prirent le contrôle du camp en 1942. Le Conseil juif créa un ontrồe du camp en 1942. Le Conseil juif créa un orchestre à cordes d'une trentaine de musiciens de très haut
niveau.
Mais c'est sous la férule du SS Albert Konrad Gemmeker, commandant de Westerbork de 1942 à 1945 et véritable amateur de musique, que la vie musicale du camp atteignit son apogée, principalement dans le domaine du cabaret. Plusieurs grandes figures de la scène berlinoise, tels Max Ehrlich, Kurt Gerron, Willy Rosen, Camilla Spira ou Erich Ziegler, obtinrent l'autorisation de monter des spectacles de cabaret d'une grande qualité professionnelle.

\section{Centres d'extermination}

La musique n'est pas absente des centres d'extermination, ainsi qu'en témoigne la présence d'orchestres ou d'ensembles à Auschwitz et Treblinka.

Auschwitz comptait deux orchestres d'hommes, l'un créé à Auschwitz I en 1942, l'autre à Auschwitz-Birkenau à la fin de la même année. Le camp des femmes d'Auschwitz disposait également de son orchestre dirigé par la violoniste Alma Rosé. Elle était la fille d'Arnold Rosé (1860-1941), violon solo de la Philharmonie de Vienne, fondateur de l'un des plus célèbres quatuors à cordes de l'époque qui portait son nom, et par ailleurs beau-frère de Gustav Mahler. Il y avait également des ensembles dans le camp tzigane et celuides familles de Theresienstadt de Birkenau, ainsi qu'au sous-campde Buna-Monowitz (AuschwitzIII). Dèslouverturedur mpde Treblinka en juillet 1942, les SS avaient formé un trio porr accompagner leurs repas et leurs soirees. Un vértable orchestre ne futcependantconstitué qual larrivee du violoniste Artur Gold, chef dun célèbre orchestre de jazz du ghetto de Varsovie. Remarqué par Kurt Franz, il fut contraint de réunir un ensemble d'une dizaine de musiciens, qui jouait à ses débuts à proximité des chambres à gaz pour couvrir les cris des victimes, puis sur la place d'appel, avec un répertoire de marches, et de chansons populaires polonaises et yiddish. L'orchestre intervint plus tard au cours de combats de boxe, de saynètes théâtrales et pour le divertissement des SS. Gold et ses musiciens furent assassinés en 1943 au cours du démantèlement du camp consécutif au soulèvement d’une partie des détenus.

\section{Orchestres des ghettos}

Les orchestres tiennent enfin une place très importante dans les ghettos, où l'administration juive dispose de coudées plus franches pour organiser une vie culturelle active. C'est le cas par exemple dans le quartier d'Amsterdam où fut progressivement installé l'ensemble des 140000 Juifs du pays avant d'alimenter les camps de transit. Le dernier Juif fut ainsi déporté en septembre 1943. Le quartier disposait d'un orchestre symphonique qui donnait ses concerts au Joodsche Schouwburg (Théâtre Juif, ancien Théâtre Hollandais) sous la direction d’Albert van Raalte.

Si le ghetto «modèle» de Theresienstadt occupe dans l'histoire de la Shoah et de la musique une place particulière, c'est dans celui de Varsovie - par ailleurs le plus important de l'Europe occupée avec ses 400000 habitants vivant dans 
des conditions de plus en plus précaires - que se développa la vie culturelle et musicale la plus active. Le ghetto comptait cinq théâtres yiddish et polonais, de nombreux clubs et cabarets, chœurs, ensembles de chambre et un orchestre symphonique.

L'Orchestre symphonique juif comportait 80 membres issus de la Philharmonie nationale, de l'Orchestre de la Radio polonaise et de celui du Grand théâtre, complété par des musiciens originaires de Berlin, Vienne et Prague déportés au ghetto. Créé en novembre 1940, il eut jusquà 1942 quatre chefs d'orchestre : Marian Neuteich, en noventeich, Adam Furmanski, Szymon Pullman, et Israel Hamerman. Le programme du premier

Le premier orchestre de Theresienstadt fut celui monté en mai 1942 par le compositeur et pianiste Carlo Taube (1897-1944), élève de Ferruccio Busoni, pour interpréter La Symphonie Terezin qu'il avait composée. Elle reflétait son expé- Karel Ancerl faisant saluer avel Haas à la fin du conaire de propagande SS de Kurt Gerron.

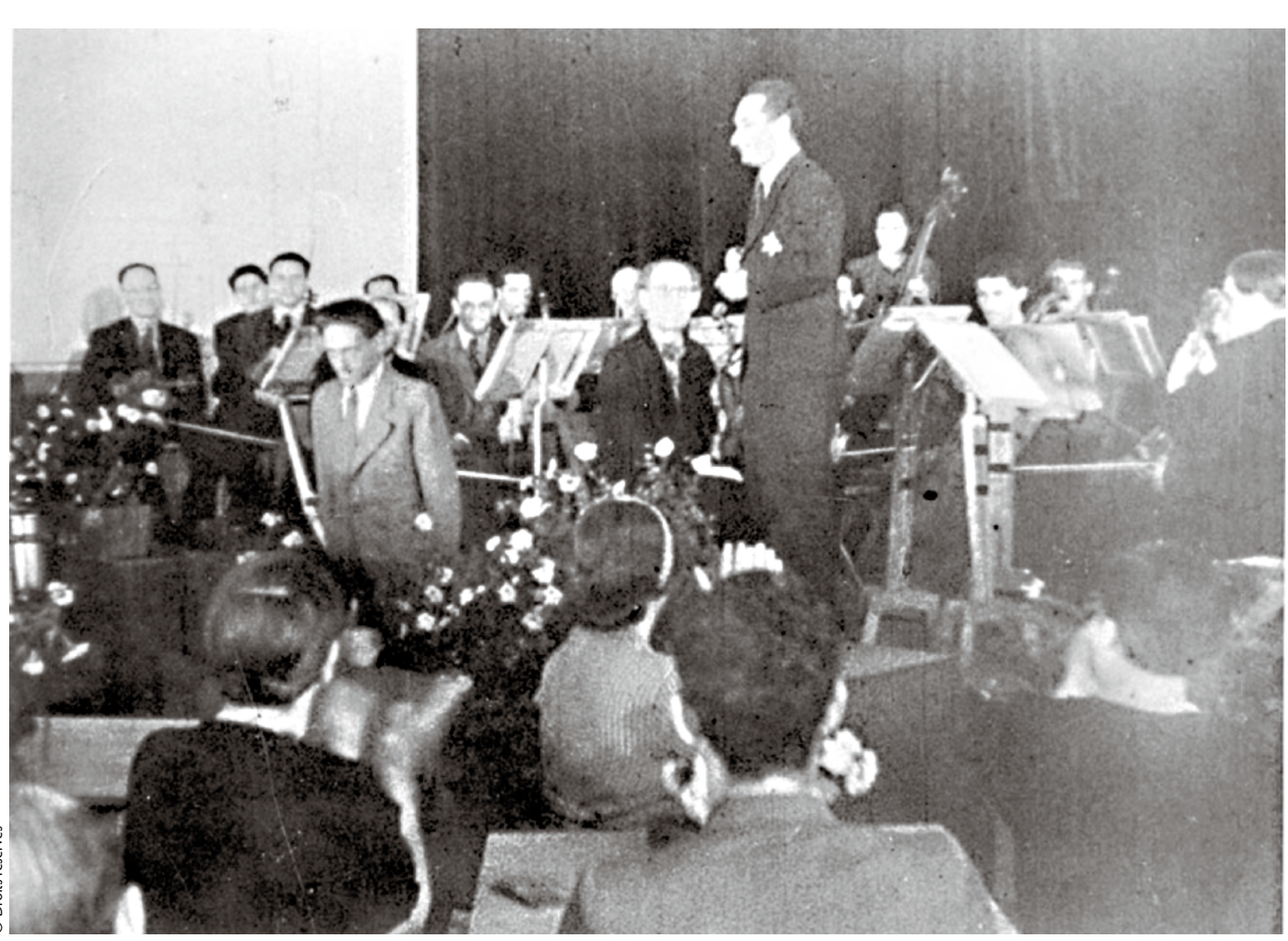

Taube, qui avait été avant-guerre pianiste dans les bars et night-clubs de Brno pour nourrir sa famille, forma plus tard un orchestre de musique légèreà Theresienstadt.Il partageait avecl' «orchestre municipal» dirigé par le danois Peter Deutsch le kiosque à musique installé sur la place principale du ghetto. Créé en 1943 et fonctionnant comme un orchestre de ville d'eau, la «Stadtkapelle» donnait des concerts quotidiens avec un répertoire de classiques favoris, et d'airs d'opéras et d'opérettes. Ses musiciens et son chef furent déportés le 28 septembre 1944 à Auschwitz-Birkenau, où les SS leur ordonnèrent de former un nouvel orchestre.

Mais la formation la plus ambitieuse fut incontestablement l'orchestre formé par le chef d'orchestre et élève de Václav Talich, Karel Ančerl, qui avaitétél'assistant de Hermann Scherchen à l’Opéra de Munich.

L'ensemble comptait environ quarante cordes, et jouait un répertoire classique avec un très haut niveau d'exécution. Il existait egalement un orchestre de chambre fondé par le violoncelliste Luzian Horwitz, et composé exclusivement de musiciens allemands, à l'exception de son violon solo Karel Fröhlich. Fröhlich avait d'ailleurs une telle réputation qu'il était violon solo de la plupart des formations instrumentales du ghetto.

La vie musicale était aussi développée dans beaucoup d'autres ghettos. Le ghetto de Łódź, qui, outre 250000 Juifs, accueillait 5000 Roms, comportait un orchestre
symphonique dirigé par Teodor Ryder (1881-1944, à Auschwitz). Avant d'être déporté symphonique dirigé par Teodor Ryder (1881-1944, à Auschwitz). Avant d'être déporté
au ghetto, ce dernier avait fait une carrière internationale, notamment à lopéra de au ghetto, ce dernier avait fait une carrière internationale, notamment à l'opéra de Lyon, puis était devenu chef d'orchestre à la Philharmonie de Łódz en 1919. De nombreux chœurs, dont celui de la Société Hazamir, des chœurs d'enfants, un théâtre de revue faisaient de Łódź un ghetto particulièrement actif sur le plan musical. La entre 1940 et 1941, accueillant plus de 70000 personnes.

Pendant l'été 1941, l'armée allemande envahit la Lituanie. Elle institua des ghettos comme ceux de Vilnius (Vilno) et Kaunas (Kovno). À Vilnius se développa une activité culturelle fortement influencée par l'idéologie sioniste du « Bund», premier mouvement socialiste juif qui y avait été fondé en 1897 et par le mouvement de résistance clandestin qui œuvrait au sein du ghetto. Un petit orchestre avait été fondé en décembre 1941 par Wolf Dumashkin, chef d'orchestre venant de Varsovie. L'orchestre donna 35 concerts pendant son existence, avec un répertoire comprenant aussi des œuvres de compositeurs « aryens ». Son premier concert, dirigé par Yaakov Gershteyn, eut lieu le 18 janvier 1942 en mémoire des Juifs assassinés. Gershteyn avait par ailleurs reconstitué son chœur d'étudiants. Un chœur hébraïque vit le

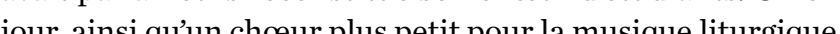
jour, ansing cheur chantant essentiellement de la musique populaire et des chansons hassidiques avec
un orchestre. 
Dans certains camps et ghettos, c'est justement par le tango que se cristallis l'expression des épreuves vécues par les détenus. Souvent écrites en yiddish, mais aussi en hébreu, roumain, russe, polonais, français, voire en allemand, ces pièces spécifiques apparurent ainsi dans les ghettos de Vilnius, Kovno, Łódź, Białystok, ainsi qu’à Auschwitz ou Dachau. Shmerke Kaczerginski, qui séchappa du ghetto de Vilnius pour rejoindre la résistance, recueillit pendant la guerre de nombreux manuscrits qu'il publia à New York en 1948 sous le titre Lider fun getos un lagern (Chants des ghettos et des camps).

\section{FONCTIONS DES ORCHESTRES}

Les fonctions des orchestres des camps étaient les suivantes :

- Rythmer la journée de travail

- Jouer pendant les punitions et les exécutions

- Donner des concerts pour les SS et les prisonniers

- Jouer pour les divertissements privés des SS

- Jouer pendant les cérémonies officielles

- Dissimuler la réalité de la vie des camps

- Accompagner le processus de sélection dans les centres d'extermination

\section{Rythmer la journée de travail}

Leur première mission était d’accompagner par des marches la sortie des commandos de travail le matin et leur retour au camp le soir. Cela permettait de compte plus rapidement le nombre de prisonniers, en rang par cinq, et le cas échéant, celui des morts rapportés le soir par leurs compagnons. Cette fonction de l'orchestre était quotidienne et ne souffrait que de rares exceptions en cas de très mauvais temps. Encore s'agissait-il plus de protéger les instruments du froid ou de la pluie que les musiciens eux-mêmes, selon le témoignage de Szymon Laks, compositeur queles directeur de lorch fonction quotidiennes s'ajoutait dans la plupart des camps celle de jouer pendant l'appel de prisonniers.

\section{Jouer pendant les punitions et exécutions de détenus}

De nombreux témoignages, documentés parfois photographiquement, attestent de la participation des orchestres des camps aux punitions : le ler janvier 1939 pa exemple, tard dans la journée, le commandant-adjoint de Buchenwald Arthur Rödl donnal'ordre de faire aligner les prisonniers, en choisit cinq qu'il fit fouetter toute la nuitaccompá parlorchestre ducamp Le 30 juillet 1942 au camp de Mauthen foren, Hans Bonarewitz futrepris après un précédé par l'orchestre du camp.

\section{Les concerts}

Ils ont lieu souvent le dimanche-puisque c'est le seul jour de repos de la semaine des détenus et des gardiens -, et réunissent en plein air sur la place d’appel (Appellplatz) ou dans les baraquements les SS et les détenus, qu'ils soient «Prominente» ou non. Cette fonction est évoquée dès les premiers camps.

Le compositeur du Dachaulied (Hymne de Dachau), Herbert Zipper, interné à Dachau d'août 1938 à 1939, décrit ainsi les concerts du dimanche dans le camp :

Concert est une expression exagérée. Nous n'avions pas de vrais instruments, mais des guitares et des instruments à cordes fabriqués par les prisonniers. Nous jouions tous les dimanches devant quelques centaines de prisonniers un programme de dix à quinze minutes comportant toutes sortes de musiques que javais écrites pendant la semaine [...] Il ne s'agissait pas toujours de musique dont jétais l'auteur. De nombreux prisonniers voulaient également entendre des pièces du genre de la Habanera de Carmen et d'autres musiques appartenant au bien commun de l'humanité.

L'orchestre du camp des hommes d'Auschwitz joua tous les dimanches de 1942 l'orchestre du camp des fent de de l'orchestre du camp des femmes de Birkenau. Les concerts étaient alors don-

\section{Les divertissements privés des SS}

Les témoignages sont nombreux pour dire que la musique jouait un rôle important dans le divertissement des SS, et que certains dentre eux en étaient grands amateurs. LeSS-Rottenführer Pery Broad, qui était chargé à Auschwitz du processus de sélection pour les chambres à gaz, était lui-même un accordéoniste virtuose qui fréquentait régulièrement le Block de l'orchestre à Auschwitz. Les orchestres et divers ensembles constitués étaient à la disposition permanente et privée des SS qui pouvaient à tout moment demander à un musicien de jouer pour eux. Toujours à Birkenau, le Dr SS Josef Mengele ou le commandant du camp Kramer venaient régulièrement écouter lorchestre du camp des femmes après une sélection.

Dans le cadre de divertissement des bourreaux, la présence du jazz, paradigme de la musique «dégénérée» dans l'idéologie nazie, ne constitue pas un des moindres paradoxes de la vie musicale des camps, alors même quả la fin de 1941, les autorités nazies durcissaient les sanctions infligées aux groupes de jeunes (Swing-Jugend) qui pratiquaient et écoutaient souvent dujazz en signe de protestation contre le pouvoir: outre une sévère correction, le contrevenant risquait de deux à trois ans d'emprisonnement, voire la déportion en camp de concentration, et la confiscation de son ses biens. L'existence de fornations de jazzà cuschwitz, Buchenwald, Mauthausen, 
l'administration de chacun des camps en question, en dit long sur la dégradation du principe même de pureté raciale au nom duquel ces autorités étaient censées agir. Le groupe de jazz d'Auschwitz semble même n'avoir fonctionné qu'au profit des orgie des SS. À Buchenwald, le Big Band «Rythmus» fut créé à lété 1943 sans autorisation, à une période où la gestion même de la vie du camp avait été laissée par les SS aux représentants des prisonniers. Son répertoire comportait des pièces de Duke Ellington, Cole Porter, Glenn Miller, W. C. Handy, Irving Berlin, Louis Armstrong Artie Shaw ou Fats Waller. Lorsqueles SS appricentlexistence de Rythmus, ilsen (lis en autorisèrent les apparitions publiques devantles autres prisonniers et lexhibèren à plusieurs reprises dans d'autres camps. Mauthausen possedait egalement une petite formation de jazz, creée elle aussi sans autorisation, qui joua a partir de l'ét 1944 pour les prisonniers. Après l'avoir entendue, les SS exigèrent que le groupe vîn animer les soirées du bordel local. Pour ne pas avoir à satisfaire cette obligation, le groupe préféra se dissoudre.

\section{L'accompagnement des cérémonies officielle}

Les orchestres jouaient également pendant les cérémonies officielles, qu'il s'agisse de l'anniversaire du Führer, lors d'inspections ou de la visite d'officiels de la SS. Certains commandants de camps fiers de leur orchestre n'hésitaient pas à l'exhiber lors de visites rendues dans d'autres camps. Szymon Laks rapporte avoi dû composer en toute hâte un hymne pour 'anniversaire du Lagerführer SS-Sturmbannführer Schwarzhuber à Auschwitz, où Heinrich Himmler fut accueilli une foi au son des trompettes d'Aïda. À Dachau en 1941, c'est une polka qui salua ce dernier.

\section{Propagande et dissimulation}

Les orchestres et de façon plus générale la musique étaient également utilisés dans le but de dissimuler aux observateurs extérieurs la réalité des camps de concentration. Cette propagande visait à tromper l'opinion internationale, ou les concentren populations habitant a proximité des camps : l'orchestre du camp d'Esterwegen où fut interné le pacifiste et opposant au nazisme Carl von Ossietzky (1889-1938), Prix Nobel de la Paix en 1936 - fut ainsi mis a contribution pour une visite de la Croix-Rouge Internationale. Le ghetto de Theresienstadt - conçu dans un but de propagande, et qui donna lieu au tournage d'un film documentaire pour montrer à quel point l'Allemagne nazie se préoccupait du sort des Juifs, reçut également une visite de la Croix-Rouge Internationale en juillet 1944.

\section{L'accompagnement du processus de sélection dans les centres d'extermination}

Cette fonction faitl'objet de controverses consécutives à des témoignages contradictoires. Le processus de sélection consistait pour les SS à déterminer à l'arrivée de chaque nouveau convoi les déportés qui seraient envoyés directement à la chambre à gaz et ceux que l'on conserverait pour exploiter leur force de travail. À Birkenau particulièrement, certains témoins attestent de la présence d'un orchestre pour accompagner cette macabre besogne, alors que d'autres la démentent catégoriquement.

Helena Dunicz-Niwińska, qui était violoniste dans l'orchestre des femmes raconte:«Les Allemands ont aussi utilisél'orchestre pour leurrer les Juifs qui étaient déportés. Ils pouvaient ainsi imaginer qu'ils avaient été amenés à Auschwitz pour travailler et que, si un orchestre jouait, c'est que on ne devait y être pas si mal.» Erika Rothschild, déportée à Birkenau, fait un récit similaire :

Ceux qui arrivaient à Birkenau étaient extraits des wagons à bestiaux et mis en rangs [...] Lorchestre accompagnait (le processus) : il était constitué des meilleurs musicien parmi les prisonniers ; selon l'origine des transports, ils jouaient de la musique populaire polonaise, tchèque ou hongroise. L'orchestre jouait, les SS distribuaient les coups, et vous n’aviez pas le temps de réfléchir... certains étaient poussés dans le camp, les autres vers les crématoires.

Louis Bannet et Shmuel Gogol, respectivement trompettiste et joueur d'harmonica dans l'orchestre des hommes, évoquent des scènes semblables.

. dans le processus de sélection:

En ce qui concerne Birkenau, je dois le démentir catégoriquement. L'orchestre ne participait pas à de tels spectacles. Je ne disculpe pas l'orchestre, je disculpe les Allemands qui aiment trop la musique pour l'utiliser dans des buts aussi prosaïques. Il nous arriva bien de jouer quelquefois sur notre podium au moment où des colonnes de condamnés marchaient, de l'autre côté des barbelés, vers les chambres à gaz, mais c'était purement fortuit, une simple coïncidence.

Anita Lasker-Wallfisch et la Française Fania Fénelon, membres de l'orchestre des femmes, contestèrent également une telle participation.

Le récit d'Helena Dunicz-Niwińska apporte probablement la réponse à ces contradictions. Elle poursuit

Durant les concerts du dimanche, nous jouions dehors, entre les baraquements. Nous interprétions des ouvertures, des marches, des airs d'opéras ou d'opérettes. Les gens descendant des wagons, avant deêtre sélectionnés, puis dirigés vers les fours crématoires, entendaient évidemment notre musique, tout comme nous pouvions les voir et avons été les témoins conscients de ce qui se passait.

Le Block de l'orchestre était situé à proximité immédiate de la rampe, et lors des concerts du dimanche en plein air programmés sans rapport avec l'arrivée des trans- 
ports, il est plus que probable que les déportés entendaient le son de l'orchestre. Le historiens pensent aujourd'hui que le processus de sélection, tout comme l'assassinat des prisonniers, n'étaient pas accompagnés - sauf exception - par de la musique. En revanche, les musiciens avaient conscience d'être entendus, et ce souvenir eut pour les survivants un impact psychologique intense.

Notons par ailleurs que pendant les derniers jours de Bergen-Belsen, les prisonniers furent forcés de porter dans les fosses communes plus de 2000 cadavres au son de deux orchestres tziganes qui se relayaient en jouant des airs de danse.

\section{ES ORCHESTRES DES CAMPS : PERCEPTION}

\section{Les orchestres vus par les prisonniers..}

Dans ce contexte oscillant entre fonctions officielles et divertissement des détenus, les musiciens avaient un statut ambivalent: membres d'orchestres voulus pa les SS, ils étaient en général soumis à un régime de travail allégé leur permettant d'une part de répéter, d'autre part d'être relativement protégés des brimades et mauvais traitements exercés par leurs gardiens. Ils bénéficiaient globalement de conditions de vie meilleures que celles des autres détenus, travaillant au chaud et recevant proportionnellement plus de nourriture.

Par rapport à la main-d’œuvre abondante des camps, qui était exploitée jusqu’à la mort pour l'industrie de guerre allemande, les bons musiciens étaient protégés par leur rareté. Aussi étaient-ils fréquemment la cible de la jalousie des autres prisonniers. Fania Fénelon raconte ainsi : «La plupart des femmes étaient trè méchantes, car nous étions privilégiées - pas à cause de la nourriture, mais nous avions un baraquement, un poêle, il ne faisait pas froid, nous étions bien habillées, alors que les autres femmes courraient pieds nus en hiver. Nous pouvions nous doucher tous les jours.» En tout état de cause, l'incorporation dans l'orchestre d'un camp signif jat une plus grande probabilité de survie, ainsiqu'n atteste Anita d'un canp sign jait une plus grande probabilite de survie, ainsi quen atteste Anit Lasker-Wallfisch dans son livre La Vérité en Héritage. Comme le rappelle Helena Dunicz-Niwińska:

Les prisonniers réagissaient de façon très contrastée. Certains d'entre eux étaien heureux de pouvoir oublier les duretés quotidiennes des réalités du camp en écoutan de la musique. D’autres prisonniers étaient scandalisés lorsque nous jouions alors que s’embrasaient les cheminées des fours crématoires. Pour eux, cétait incompréhensible. Nous aussi les musiciennes, nous vivions cette expérience de façon très différente. Certaines d'entre nous étaient effondrées de donner des concerts dans de telles conditions.

Les avis divergent sur le rôle que joua la musique sur le moral des prisonniers dans les camps. Pour certains, elle représente un moyen de détente, d'oubli des terribles conditions de vie. Pour d'autres, elle n'eut pas ou peu d'effet.
Dans son livre de souvenirs sur son internement Musiques d'un autre monde publié en 1948, Szymon Laks s'exprime ainsi avec force contre toute vision «idéalisée» de la fonction de la musique dans les camps :

Indépendamment de ce que jai vu et entendu moi-même, jai recueilli des informations auprès de mes amis et relations vivant éparpillés dans le monde entier, qui furent détenus dans divers camps de concentration. Ils sont presque tous de l'vis que ne furent exclusivement produites dans les camps que des chansons vilgaires à

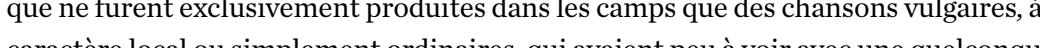
caractère local ou simplement ordinaires, qui avaient peu à voir avec une quelconque force dâme humaine [...] nl est désagréable de détruire les légendes. Mais il est encore plus désagreáable de lire que la musique était un médicament pour lâme malade du détenu «précisément dans les conditions concentrationnaires». Car ce n’est pas vrai [...] En réalité, et à peu d'exceptions près, la vraie nature d'un détenu se montrait sous l'influence de la faim, des coups et de la maladie : la nourriture et de vrais médicaments soignaient son âme malade, pas la musique !

Il est vrai que Laks porte ici un regard de professionnel sur la qualité intrinsèque des morceaux joués dans les camps.

\section{Et par les bourreaux}

\section{Laissons parler Helena Dunicz-Niwińska :}

Les Allemands voulaient que l'on joue le dimanche. Je ne peux pas dire que nous jouions seulement pour eux. Il s'agissait de «concerts du dimanche» pour les prisonniers, mais les Allemands venaient aussi écouter. Les SS du bureau du commandant venaient aussi assister à nos répétitions, pas dans leur totalité, mais pour un petit moment. Par exemple, le commandant du camp des femmes, Hoessler Maria Mandl [...] Même le Docteur Mengele rendit visite à notre Block Les concerts du dimanche

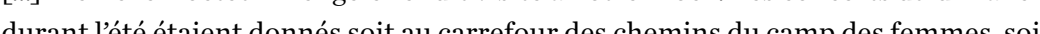
dura los soit entre les Blocks de lhopital. En hiver nous jorions dans un des Bocks de hôt. Alma Rosé faisait tout pour que notre niveau soit le meilleur possible. Elle travaillait énormément. Nous avions conscience que nous aidions les Allemands non seulement en rendant plus facile la marche des Kommandos de travail, mais aussi par le fait quà l'époque où Alma Rosé dirigeait l'orchestre, le niveau était tellement élevé, que les Allemands étaient tout simplement heureux d'avoir un tel orchestre dans le camp des femmes. Cela n’arriva jamais ailleurs. Ils en étaient fiers, et s'en vantaient. On nous exhibait aux personnes extérieures qui venaient contrôler le fonctionnement du camp.

En donnant pour quelques heures aux SS le sentiment d'une vie «normale», les concerts et les orchestres contribuaient involontairement à l'effort de guerre nazi. Par ailleurs, les orchestres étaient très souvent un enjeu de pouvoir entre chefs de 

bitions dans d'autres camps voisins.

\section{DU BON USAGE DE LA MUSIQUE CONCENTRATIONNAIRE}

Au printemps 2009, l'Ensemble Voix Étouffées formé par l’auteur des présentes lignes collaborait pour la première fois de sa jeune existence avec le Musée d’Au-

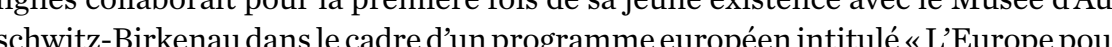
sché les citoyens ». Depuis sa création en 2003, l'Ensemble s'était spécialisé dans la redécouverte de compositeurs victimes du nazisme et de leurs œuvres, regroupées sou le vocable générique que leur avait donné les nazis, celui de «musique dégénérée (entartete Musik)». Il était inévitable que ses pas croisent un jour la musique issue directement des camps de concentration, d’où sa présence à Auschwitz un beau jour d'avril 2009 pour jouer des œuvres sur le lieu même du supplice de leurs compositeurs. Dans l'impossibilité pratique et éthique qu'il y avait à présenter ce concert dans le camp lui-même, les relations publiques du Musée avaient organisé l'événement à l'école de musique d'Oswiecim, et avaient organisé une rencontre entre notre groupe et des professeurs de la région de Petite Pologne dans le cadre d'un programme de formation des enseignants à l'histoire de la Shoah.

Cette expérience se reproduisit depuis lors à plusieurs reprises - six pour être précis -,impliquant de plus en plus les élèves de lécole de musique, et notamment sa chorale, et leurs professeurs, ainsi qu'un groupe d'étudiants d'une école d'arts appliqués parisienne, l'ENSAAMA. Dans les programmes de ce qui était devenu un échange franco-polonais régulier figuraient par exemple l'opéra pour enfants Brundibár de Hans Krása (1899-1944), joué plus de 50 fois à Theresienstadt de 1942 à 1944 avant la déportation vers Auschwitz du compositeur et de ses petits chanteurs, des mélodies de Szymon Laks (1904-1972) qui fut un temps directeur de l'orchestre des hommes d'Auschwitz I ou des mélodies d'Ilse Weber(1902-octobre 1944 - assassinée à Auschwitz) arrangées à nouveau pour la chorale de l'école. Notre toute première a Aushoreme collaboration avait éte une double révelation, celle du lieu et de son esprit, mais auss

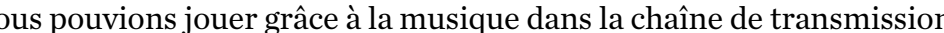
de l'histoire de la Shoah.

Depuis lors, le Forum Voix Étouffées a développé en son sein une forte activité pédagogique en collaboration avec des lieux de mémoire de la déportation, comme le Musée du Struthof, celui du Camp des Milles, le Cercil dans le Loiret, le Mémoria d'Alsace-Lorraine à Schirmeck, ou le Mémorial de Langenstein-Zwieberge en Saxede témoins directs, comme celle d'Eva Hermannová qui chanta dans le chœur de Brundibár à Theresienstadt. D'autres ateliers se sont développés avec des orgeni作 列 cette dernière portant sur la formation des enseignants pour aborder la déporta- tion et la Shoah par la musique. Des collaborations se sont récemment ouvertes avec plusieurs branches régionales du Réseau Canopé du ministère de l'Éducation nationale français, permettant de toucher de nombreux établissements scolaires d'enseignement général. Les jeunes musiciens ne sont pas absents de nos préoccupations par un travail fait dans le cadre d'un réseau européen réunissant une quinzaine de pays de l'UE et divers orchestres de jeunes avec lesquels est abordé et mis en perspective historique et politique ce répertoire. L’Ensemble Voix Étouffées est par ailleurs régulièrement invité à la Commission Européenne à Bruxelles, ou est par ailleurs régulièrenent invé à la Conssón dans de nombreux pays europeens - Lituanie, Macédoine, Bulgarie, Autriche, Allemagne... - pour commémorer par la musique la déportation dans son ensemble et
la Shoah en particulier.

Au fil du temps, et par-delà ses aspects les plus positifs, cette activité militante suscite cependant de nombreuses interrogations, voire metà jour des contradictions d’ordre déontologique, scientifique, politique et musical, qui nous forcent à réfléchir sur ce que l'on pourrait appeler «les bonnes pratiques» de la mémoire, tout comme Georges Bensoussan parle «du bon usage» de cette dernière.

Présenter au public un répertoire d'œuvres composées pendant l'internement de leur auteur - voire exécutées par les orchestres constituant le sujet de cet article leur auteur - voire exécutees par les orchestres constituant le sujet de cet article dans le systeme concentrationnaire nazi n'est pas sans risque. Parce qu'un tel travail met en jeu des disciplines croisees - outre la musique interviennent l'histoire, la germanistique, la philosophie, les sciences politiques et bien d'autres - et que dans chacune de ces disciplines, la vérité se trouve dans le détail, on ne peut traiter de ces sujets qu'avec prudence et compétence, et non de façon forfaitaire. La «musique des camps » à laquelle on a souvent à faire face dans les concerts afférents, et qui est prétexte à mélanger dans un même programme des œuvres d'Olivier Messiaen, prisonnier de guerre protégé par la Convention de Genève, et celle d'Ullmann, assassiné à Auschwitz à cause de ses origines juives, est un concept non seulement assassins mais au'on pourrait ausiment en les confont étrangeres les unes aux autres : la guerre n'est pas juridiquement un crime contre l'Humanité, alors qu'un génocide l'est. J'ai bien conscience que la plupart du temps, de tels projets sont plus le fruit de l'ignorance que de la malhonnêteté intellectuelle, mais la première n'est pas une excuse. Ce genre d'approximation historique alimente une «Shoah-business» dont les ressorts exploitant une vision purement compassionnelle des événements sont pédagogiquement inopérants et déontologiquement plus que contestables.

Une autre difficulté consiste à affronter la contradiction suivante : le concept de musique concentrationnaire recouvre en réalité plus une sociologie qu'un art, un musique conce en réalité plus un terme. Parmi le très petit nombre d’ouvres dontl'intérêt musical dépasse le contexte 


\section{DOSSIER}

Les orchestres dans le système concentrationnaire nazi : fonctions et réception (suite) de leur élaboration, celles qui sont venues jusqu’à nous rentrent dans le répertoire musical «normal » précisément parce qu'elles ne renvoient pas directement à l'univers concentrationnaire et son cortège d'ombres, mais à cause de leurs qualités artistiques intrinsèques. Brundibár par exemple, qui prend aujourd'hui en toute justice la place qui lui revient dans le répertoire de la musique pour la jeunesse - et qui d'ailleurs n’a pas été composé à Theresienstadt, mais à Prague en 1938 -, est un excellent outil de transmission de la Shoah parce qu'il s'agit d'abord et avant tout d'une œuvre de qualité qui permet d'être abordée à la fois comme œuvre musicale, et comme témoin des événements qui accompagnèrent son rôle à Theresienstadt.

La vie musicale dans les camps nazis n'est qu'une partie des immenses dommages causés à la culture européenne par le régime hitlérien, et le travail mené depuis quelque vingt ans pour faire revivre ce que ce dernier a détruit est une tâche difficile dans un monde où la mémoire est constamment invoquée, mais fort peu construite. Pour valoriser ce répertoire perdu, il faut prendre - Brundibár école de musique Auschwitz. garde à ne présenter que des chefs d'œuvre susceptibles de rivaliser avec ceux du répertoire «commun». Toute autre approche risque de conduire à jeter le bébé avec l'eau du bain, et ce n'est rendre service à personne que de présenter des pièces médiocres sous prétexte que leur auteur a péri victime du nazisme.

La sensibilité afférente à la musique doit s’accompagner dans ce domaine d'une grande exigence scientifique et historique pour aider à construire une mémoire «positive» et constructive des désastres causés par le nazisme.

Amaury du Closel est compositeur, chef d'orchestre. II a créé le Forum Voix Étouffées qui contribue depuis 2002 à la diffusion de la musique de compositeurs victimes du nazisme et qui organise de multiples ateliers pédagogiques. II est l'auteur entre autres de «Entartete Musik». Musiques interdites sous le III Reich (2015). 